\title{
Research on Dimensions of Risk Perception of Genetically Modified Food by Consumers
}

\author{
Zeng Zhi, Xiang Gaoyue, Zheng Shichen, Shen Yongjian \\ Nanjing University of Chinese Medicine, College of Economics and Management, Nanjing, China \\ Email address: \\ zengzhinju@163.com (Zeng Zhi)

\section{To cite this article:} \\ Zeng Zhi, Xiang Gaoyue, Zheng Shichen, Shen Yongiian. Research on Dimensions of Risk Perception of Genetically Modified Food by \\ Consumers. Psychology and Behavioral Sciences. Vol. 5, No. 4, 2016, pp. 113-116. doi: 10.11648/j.pbs.20160504.16
}

Received: June 1, 2016; Accepted: July 6, 2016; Published: July 11, 2016

\begin{abstract}
Currently, the researches and development about transgenosis are witnessing a strong momentum across the world, and its industrialization enjoys rapid development as well. Moreover, in China, developing transgenosis also serves as a crucial strategic decision for the Central Committee of the Chinese Communist Party and State Council. Sufficient evidences have shown that genetically modified food (GMF) is safe, but risk perception of GMF by consumers turns out an important factor that influences its commercialization. Adopting the method of questionnaire survey and taking GMF as research object, this paper discusses the dimensions of consumer risk perception, including health risk, function risk, socio-psychological risk, economic risk and time risk, and finds out that consumers pay more attention to the health risk and economic risk of GMF.
\end{abstract}

Keywords: Genetically Modified Food, Risk Perception, Dimension

\section{Introduction}

In 2016, the No.1 Document of Central Government in China proposed to "enhance research and development of and supervision over agricultural transgenosis technology and conduct promotion prudently on the basis of safety", making GMF redraw public attention and triggering heated debates. In 1997, the commercialized plantation of GM cotton was approved in China. By 2015, the plantation area of GM cotton had reached 50 million mu. In 2008, major special project for transgenosis were set up, and remarkable breakthroughs were achieved: the number of cloned important genes reached up to 137, with 1036 patents obtained, and specialized application of transgenosis had enjoyed rapid development. The biggest doubt that consumers have about GMF lies in safety, hence inviting panic comments--for example, GMF can cause infertility. Repeated unreal public views make consumers unable to understand the issue objectively, so they keep increasing their risk perception of GMF. Consumer risk perception includes judgment upon food safety, mood and attitude, which can interpret the mentality and potential behavior of consumers against GMF [1]. From the angle of risk perception, it is significant to study mentality and behavior of consumers against GMF and to manage the risks of GMF as well in theoretical and practical terms. This paper, with GMF as the research object, focuses on the dimensions of risk perception in purchase, so as to provide a reference for faster and better development of GMF industry.

\section{Literature Review}

\subsection{Concept of Risk Perception}

Risk perception is not only reflected by digital numbers, but have a close relationship with our psychology, social and culture [2]. In 1960, Raymond Bauer at Harvard University derived the concept of risk perception from psychology to study consumer behavior. In purchasing, consumers sometimes can't prejudge the correctness of their purchasing behavior and thus the purchasing result may be not satisfactory, so consumers are faced with risks. Risk perception includes uncertainty of decision and seriousness of result [3]. Bauer held that perceived risk was subjective, and consumers would only handle and respond to their perceived risks. Later on, scholars conducted in-depth researches on risk perception and kept improving the concept. Cox thought that consumers' behavior was targeted, and when consumers were not sure whether the consumption 
would satisfy the target, risk perception came into being. Cox defined risk perception as the function of the two factors, namely impossibility of producing unfavorable consequences and the loss perceived by consumers. On the basis of empirical studies, Cunningham held that risk perception was mainly influenced by two factors, namely seriousness of unfavorable consequence brought by consumption and probability of unfavorable event occurrence. If consumers hold that the uncertainty and unfavorable consequence are serious, then the risk perception would be high. His definition has been recognized by most scholars [4]. ÇABUK held that risk perception is the uncertainty of unpredictable consequences when consumers choose to buy [5]. Through the literature on risk perception, it can be known that the concept of risk perception includes two important factors, namely uncertainty and seriousness of consequence. This paper defines risk perception as possible loss and risk borne by consumers in the process of consumption.

\subsection{Dimensions of Risk Perception}

Dimensions of risk perception serve as a focus in studying risk perception. In first introducing the concept of "risk perception", Bauer did not point out the category and content of it. On the basis of verifying risk perception as a multidimensional variable, scholars hold that risk perception possesses different dimensions. In 1967, Cunningham pointed out that perceived risk included capital loss, physical loss, social consequence and time loss, etc. In 1968, Woodside thought that risk perception had three dimensions, i.e., economic risk, function risk and social risk. In 1971, Roiselius pointed out four kinds of risk possibly facing consumers in purchasing, namely economic risk, time risk, psychological risk and physical risk. Jacoby proposed the five dimensions of risk perception, namely physical risk, social risk, functional risk, psychological risk and financial risk. Later, Stone and Gronhaug developed six-dimension risk perception by adding time risk five ones. Their studies indicated that six-dimension risk perception could explain $88.8 \%$ of risk perceptions, and financial risk, physical risk, function risk, time risk and social risk all influenced psychological risk. Mowen proposed that risk perception included performance risk, financial risk, social risk, time risk, entity risk, socio-psychological risk and opportunity cost risk. Most current literature agrees with the six dimensions of Stone and Gronhaug. Domestic scholars have also studied risk perception dimensions. For different research objects, there are different structural dimensions. Wang Jiayi used weight analysis and independent sample $\mathrm{T}$ test method to propose college students' risk perception dimensions of dairy products, including health risk, function risk and psychological risk [6]. Lv Yanfen pointed out that risk perception of green food consumption could be divided into six dimensions, namely opportunity cost, emotion, time, finance, physical and mental safety as well as function risk [7]. Li Nan proposed that consumers' risk perception of healthcare products included socio-psychological risk, product risk and health risk [8]. GMF as a controversial product at present, different scholars have proposed different dimensions for GMF. Qing Ping and Wu Yue et al. held that consumers' risk perception of GMF included political risk, time risk, financial risk, environmental risk, health risk and descendant risk [9]. Feng Liangxuan held that GMF risk perception included performance risk, environmental safety risk, physical health risk and socio-economic risk [10]. Chen Congjun thought that GMF risk perception covered five dimensions of social trust, food safety, GMF knowledge, GM technology and health concern [11]. Based on current literature and pre-survey, this paper proposes that consumers' risk perception of GMF mainly includes health risk, economic risk, time risk, function risk and sociopsychological risk.

\section{Questionnaire Design and Survey}

Based on current literature, the preliminary questionnaire of risk perception was designed, and then experts were consulted for guidance. On this basis, consumers were contacted for interview and pre-survey. The number of presurvey questionnaires was 30 , with the valid being 30 . According to pre-survey result, corrections were made to the questionnaire to obtain the final one.

Questionnaire for risk perception mainly included three parts: part one introduction to present the purpose of questionnaire, part two personal basic information and part three surveying issues related with GMF risk perception dimensions. The questionnaire design adopted Likert Scale by choosing "absolutely disagree, disagree, neutral, agree, absolutely agree" to show consumers' risk perception. One item was chosen by the respondents, with data statistics being respectively " $1,2,3,4,5$ " for differentiation. Convenient samples were chosen as survey respondents, i.e., Nanjing citizens; and random surveying was adopted, i.e., survey respondents were searched in supermarkets and living quarters for site filling questionnaires to be retrieved on the spot. In total 340 questionnaires were handed out, among which the valid was 294 after removing those with too many omissions and willful filling, reaching a validity rate of $86.4 \%$. Data collected by survey questionnaires were analyzed with SPSS20.0 software to obtain research conclusions.

\section{Questionnaire Result Analysis}

\subsection{Sample Statistical Features}

According to the data collected by questionnaire, among the 294 questionnaires, male consumers accounted for $39.5 \%$ and female consumers accounted for $60.5 \%$. In terms of age, the number of consumers $\leq 29$ years old was 72 ; the number of consumers 30-39 years old was 96 (the highest); the number of consumers 40-49 years old was 93; the number of consumers 50-59 years old was 33 (the lowest). The number of consumers with an education of middle school or under was the lowest, only registering a number of 32; the number of consumers with an education of high school and professional training was 53; 
the number of consumers with a graduate education was 153 , accounting for $52 \%$ of the total; the number of consumers with a postgraduate education was 56 . The number of colleges and universities in Nanjing is huge, so the educational level is basically high. The survey respondents in this research could be divided into 5 layers: consumers with an income of 40005999 hold the largest proportion, accounting for $39.1 \%$ and those with an income of $\leq 2000$ and $\geq 8000$ were less. The survey respondents conformed to basic reality of Nanjing and consumption of GMF. Therefore, it carried practical significance. Sample details are shown in Table 1.

Table 1. Demographic Variable Description of Survey Subjects.

\begin{tabular}{llll}
\hline & Demographic Feature & Frequency & \% \\
\hline Gender & $\mathrm{M}$ & 116 & 39.5 \\
\multirow{3}{*}{ Age } & $\mathrm{F}$ & 178 & 60.5 \\
& $\leq 29$ & 72 & 24.5 \\
& $30-39$ & 96 & 32.7 \\
& $40-49$ & 93 & 31.6 \\
Education & $50-59$ & 33 & 11.2 \\
& Middle School and Under & 32 & 10.9 \\
& High School and Professional & 53 & 18.0 \\
& Training & 153 & 52.0 \\
& Graduate Education & 56 & 19.0 \\
& Postgraduate Education & 12 & 4.1 \\
& $\leq 2000$ & 72 & 24.5 \\
& $2000-3999$ & 115 & 39.1 \\
& $4000-5999$ & 55 & 18.7 \\
& $6000-7999$ & 40 & 13.6 \\
\hline
\end{tabular}

\subsection{Questionnaire Reliability and Validity Analysis}

Reliability refers to the stability and dependability of a questionnaire. As for the same questionnaire, the surveys by the same batch of respondents will produce the same results, and highly reliable questionnaire can truly reflect the attitude of consumers. This paper uses Cronbach's Alpha coefficient to measure the internal consistency of questionnaire. The higher the reliability, the larger the $\alpha$ coefficient. When the $\alpha$ coefficient is larger than 0.9 , the reliability of the questionnaire is very high; when $\alpha$ coefficient is larger than 0.7 , the questionnaire data is reliable, while 0.7 serves as the standard for reliability. When $\alpha$ coefficient is $\leq 0.6$, the internal consistency of the questionnaire is poor. After collecting questionnaires, SPSS20.0 was adopted to analyze reliability (as shown in Table 2). The $\alpha$ coefficient for 15 items of GMF risk perception was 0.725 , indicating that the internal consistency of survey data was sound and respondents' attitude can be well reflected.

Table 2. Questionnaire Reliability Test.

\begin{tabular}{lll}
\hline Variable & Cronbach's Alpha Coefficient & Survey Item \\
\hline Risk Perception & 0.725 & 15 \\
\hline
\end{tabular}

Validity refers to the degree of survey results in reflecting the matters to be measured. This paper adopted explorative factors in SPSS to conduct analysis of validity. Through KMO and Bartlett Sphericity Test, the validity test was completed. When KMO value is $\geq 0.9$, it means that it is very suitable for factor analysis; when $\mathrm{KMO}$ value is $\geq 0.8$, it means that it is suitable for factor analysis; when KMO value is $\geq 0.7$, it means that it can perform factor analysis. Test results showed that the KMO value was 0.746 and the $P$ value of Bartlett Sphericity Test was $<0.001$, indicating a high validity of the questionnaire.

Table 3. KMO and Bartlett Test.

\begin{tabular}{ll}
\hline Sampling of enough Kaiser-Meyer-Olkin measurement & .746 \\
Bartlett Sphericity Test Chi-Squared Approximation & 2524.985 \\
df & 105 \\
Sig. & .000 \\
\hline
\end{tabular}

\subsection{Factor Analysis of Risk Perception}

It is shown by KMO measure and Bartlett Sphericity Test that factor analysis can be conducted of samples. This paper mainly adopted principal component analysis by extracting factors for orthogonal rotation. As shown in Table 4, 5 factors can be extracted, namely there are 5 dimensions to GMF risk perception. According to the meaning of items, they can be summed up into health risk, function health, sociopsychological risk, economic risk and time risk. According to Table 5, the total explaining variance of 5 extracted factors is $80.035 \%$, indicating that the 5 factors enjoy great interpreting power. Health risk refers to the possible unfavorable influence of GMF upon health; economic risk refers to the possible extra expenditure caused by unsafety of GMF; time risk refers to the possible extra time investment into the safety of GMF; function risk refers to the possible absence of nutritional supplementation of GMF; socio-psychological risk refers to the possible anxiety caused to others by GMF.

Table 4. Rotating Element Matrix.

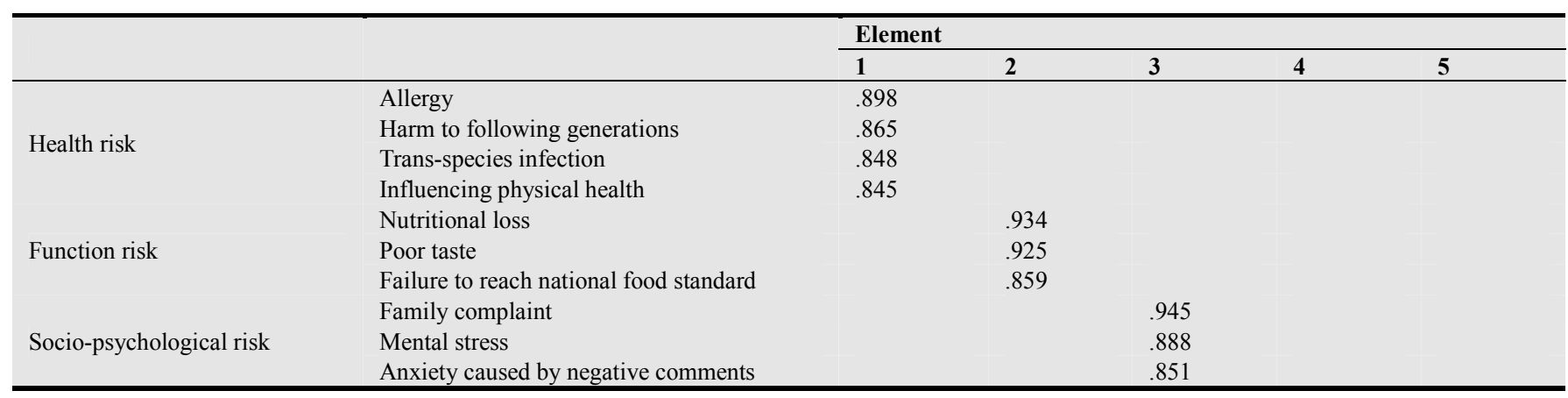




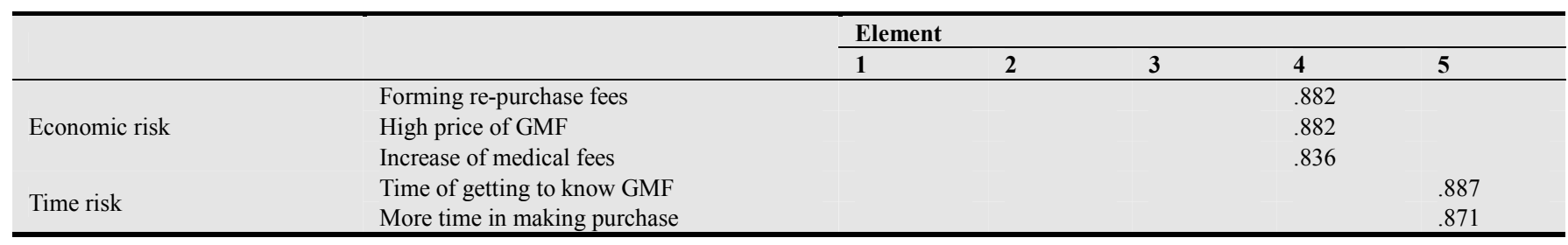

Extracting method: principal components; rotating method: orthogonal rotation of Kaiser; rotation restrains after 5 times of iteration

Table 5. Total Variance Explaining Matrix of Factors in Risk Perception.

\begin{tabular}{llllllllll}
\hline \multirow{2}{*}{ Element } & \multicolumn{3}{c}{ Initial feature value } & \multicolumn{3}{c}{ Extracting square and loading } & \multicolumn{3}{c}{ Rotation square and loading } \\
\cline { 2 - 10 } & Total & Variance & Accumulative & Total & Variance & Accumulative & Total & Variance & Accumulative \\
\hline Health risk & 3.479 & 23.195 & 23.195 & 3.479 & 23.195 & 23.195 & 3.044 & 20.295 & 20.295 \\
Economic risk & 3.224 & 21.491 & 44.687 & 3.224 & 21.491 & 44.687 & 2.555 & 17.033 & 37.328 \\
Function risk & 2.221 & 14.807 & 59.493 & 2.221 & 14.807 & 59.493 & 2.496 & 16.641 & 53.969 \\
Socio-psychological & 1.763 & 11.750 & 71.243 & 1.763 & 11.750 & 71.243 & 2.310 & 15.399 & 69.369 \\
risk & 1.319 & 8.791 & 80.035 & 1.319 & 8.791 & 80.035 & 1.600 & 10.666 & 80.035 \\
time risk & & & & & & &
\end{tabular}

Extracting method: principal components

\section{Conclusion}

This paper selects GMF as research object, utilizes empirical analysis method to discuss the 5 dimensions possessed by risk perception of customers for GMF, namely health risk, function risk, socio-psychological risk, economic risk and time risk, which can explain $80.035 \%$ of the total variance. From the 5 factors extracted, it can be discovered that health risk and economic risk can respectively interpret $23.195 \%$ and $21.491 \%$ of the total variance respectively, $44.687 \%$ altogether, indicating that consumers pay more attention to the influence of GMF upon health as well as economic input. Government should formulate scientific supervision schemes to uplift the risk evaluation, guidance and monitoring ability over GMF, publish relevant test standards and results of GMF, and make information of GMF transparent, so as to allow consumers to keep risk perception of GMF within a reasonable scope. Market supervision for GMF should be enhanced so as to ensure legal and standard operation of GMF market. In addition, a reasonable price should be set for GMF to highlight its price advantage, and meanwhile guarantee work should be done well to protect consumers.

\section{Acknowledgements}

This paper is the research results of "the philosophy and social science research project (Project Number: 2013SJB6300076)" in the Department of education of Jiangsu province.

\section{References}

[1] Guo Xuesong, Tao Fangyi, Huang Jie. Food Risk Perception Studies of Urban Residents: A Case Study of Rice Consumption in Xi'an [J]. Beijing Social Sciences, 2014(11): 19-28.
[2] Aleksejeva I. Genetically Modified Organisms: Risk Perception and Willingness to Buy GM Products [J]. Management theory and studies for rural business and infrastructure development, 2012, 4(33): 5-9.

[3] Bauer R A. Consumer Behavior as Risk Taking [J]. Dynamic Marketing for a Changing World, 1960, 398.

[4] Zhou Weina. Research on Influence of Risk Perception in Wechat Payment upon Use Intention [D]. Harbin Institute of Technology, 2015.

[5] ÇABUK S, TANRIKULU C. The Role of Perceived Risk, Uncertainty Avoidance, and Innovativeness in Willingness-ToBuy Genetically Modified Foods [J]. Cag University Journal of Social Sciences, 2014, 11(1).

[6] Wang Jiayi. Explorations into Dimensions Influencing Risk Perception of College Students of Food [D]. Jilin University, 2012.

[7] Lv Yanfen. Empirical Studies of Dimensions in Green Food Risk Perception [J]. Tianjin Agricultural Sciences, 2011(3): 77-80.

[8] Li Nan. Research on Consumers' Risk Perception of Health Care Food [D]. Southwest Jiaotong University, 2015.

[9] Qing Ping, Wu Yue. Empirical Analysis of Consumers' Risk Perception of GMF [J]. Influence of Science upon Society, 2010(02): 38-41.

[10] Feng Liangxuan. Research on Public Cognition of Risk in GMF [D]. Huazhong Agricultural University, 2013.

[11] Chen Congjun, Sun Yangxue, Liu Jundi. Analysis of Factors Influencing Consumers' Risk Perception of GMF [J]. Journal of Northwest Agro-forestry University of Science and Technology (Social Sciences), 2015(04): 105-110. 